\title{
A rare case report of congenital high airway obstruction syndrome presenting in a 23 weeks foetus
}

\author{
Madurai Padmanabhan Kanchana*, Muthu Prabha Sellamuthu, Revathy Mahendran
}

Department of Pathology, Institute of Obstetrics and Gynecology, Madras Medical College, Chennai, Tamil Nadu, India

Received: 12 January 2018

Accepted: 10 February 2018

*Correspondence:

Dr. Madurai Padmanabhan Kanchana,

E-mail: mpkanchana@rediffmail.com

Copyright: (c) the author(s), publisher and licensee Medip Academy. This is an open-access article distributed under the terms of the Creative Commons Attribution Non-Commercial License, which permits unrestricted non-commercial use, distribution, and reproduction in any medium, provided the original work is properly cited.

\begin{abstract}
Congenital High Airway Obstruction Syndrome or CHAOS is a blockage of the foetus's trachea or larynx due to many factors including narrowing of the airway, a web-like membrane or even tracheal atresia. In the uterus, the foetal lungs constantly produce fluid and as a result of this airway blockage in the trachea, the lung fluid cannot escape out of the foetal mouth. Because of this the foetus's lungs become distended with fluid and over distended lungs can put pressure on the heart and affect the heart's ability to function. If the heart cannot function effectively hydrops or congestive heart failure can occur. We present a rare case of CHAOS diagnosed prenatally at about 23 weeks by USG in our hospital.
\end{abstract}

Keywords: Flattened diaphragm, Hyper inflated enlarged lungs, Laryngeal atresia

\section{INTRODUCTION}

Congenital high airway obstruction sequence (CHAOS) is a rare life-threatening condition characterized by complete or near complete intrinsic obstruction of the foetal airway. ${ }^{1}$

Till 2007 only 52 cases have been reported. ${ }^{2}$ This clinical condition was brought into notice firstly by Hedrick in the year $1994 .^{3}$ Congenital high airway obstruction syndrome (CHAOS) is defined as complete or partial obstruction of the foetal upper airways. CHAOS is usually caused by atresia or stenosis of the larynx or trachea. The true incidence of CHAOS is unknown. If the syndrome is unrecognized during the prenatal period, it usually results in stillbirth or death shortly after delivery. ${ }^{2,4}$ Diagnosis can be possible as early as 15 weeks of gestation. The prenatal ultrasound findings are: large echogenic lungs, flattened or inverted diaphragms dilated distal airways and foetal ascites or hydrops. If foetus has a tracheo-oesophageal fistula the characteristic findings on USG may be absent. ${ }^{2}$ Earlier diagnosis of CHAOS is important as new evolving treatment modality called EXIT (ex utero intrapartum treatment) procedure is available. $^{2}$

\section{CASE REPORT}

A 21-year-old primi gravida was referred for a routine second trimester antenatal ultrasound at 23-week gestational age. Other routine laboratory investigations were found to be normal. Ultrasound examination revealed bilateral enlarged and echogenic lungs with prominent fluid filled trachea, bronchi and bronchioles up to larynx and also with post stenotic dilation of larynx and distal airway due to laryngeal atresia.

The diaphragm was inverted with displacement of heart, foetal ascites and polyhydramnios was present. No other genetic analysis was done. 


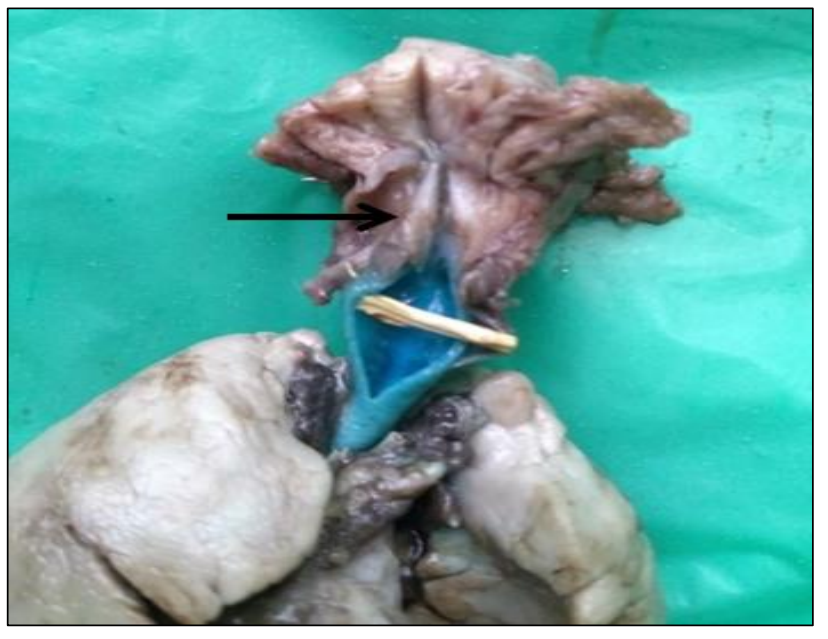

Figure 1: Airway obstruction in the glottis level, no dye above the level of glottis.

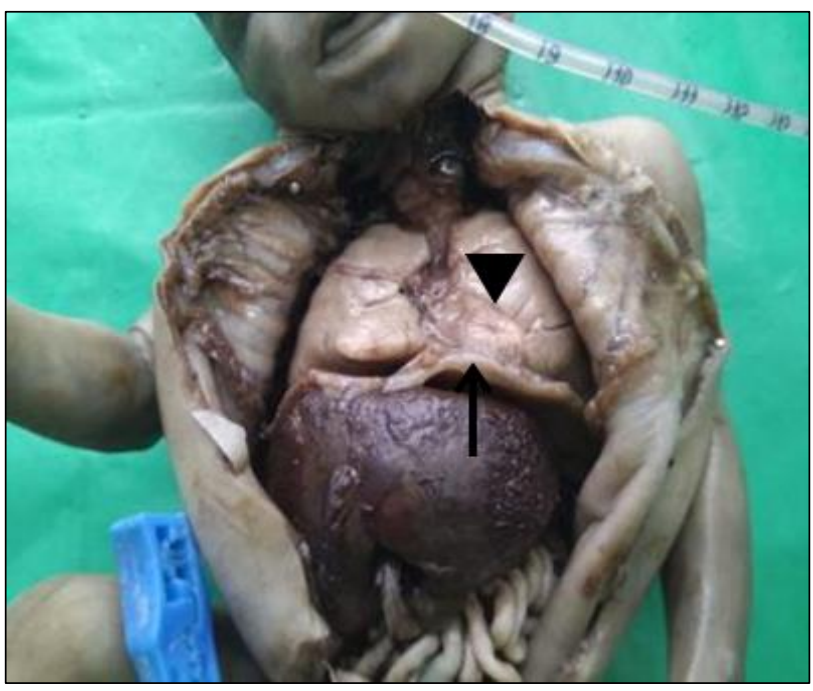

Figure 2: Displaced heart and diaphragmatic flattening.

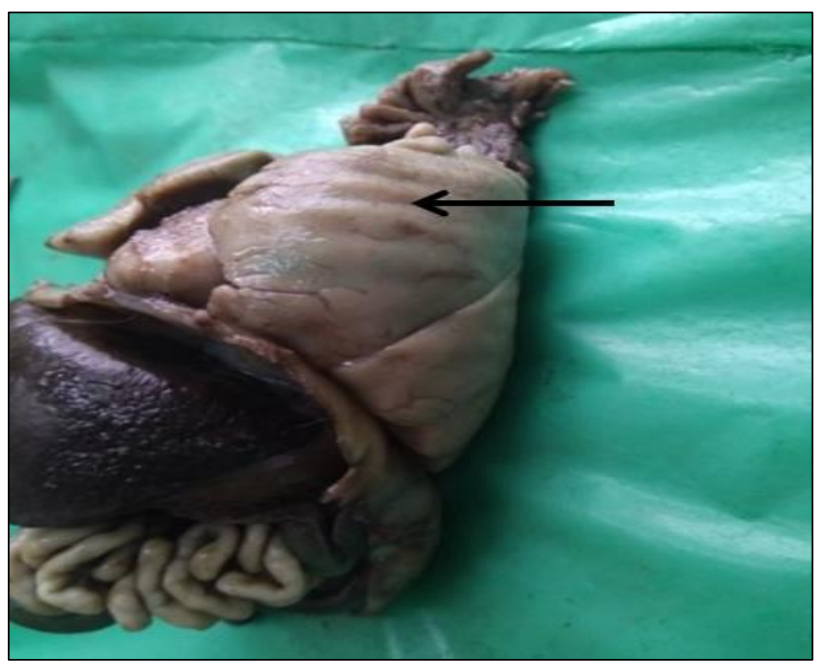

Figure 3: Voluminous lung with costal impression on external surface.
Based on ultrasound, diagnosis of CHAOS was made. The parents were counselled regarding the relatively poor prognosis of the syndrome and the pregnancy was terminated according to the choice of the family. Foetus was submitted to Pathology Department for foetal autopsy and for confirmation of the diagnosis.

Foetus weighed 500 grams with attached umbilical cord measuring $5 \mathrm{~cm}$ length. The foetal anthropometry showed crown rump length $20 \mathrm{~cm}$, head circumference $20 \mathrm{~cm}$, chest circumference $18.5 \mathrm{~cm}$ and abdominal circumference $20 \mathrm{~cm}$. External examination showed normal facial appearance, normal external genitalia and spine. Abdomen was distended, and the anus was found to be patent. Congenital abnormalities associated with other syndromes were not seen.

Foetal autopsy was done by putting an inverted Y shaped incision and the radiological findings were confirmed. The internal examination showed diaphragmatic inversion, voluminous lungs, displaced heart and foetal ascites. To confirm laryngeal stenosis a dye was injected from trachea and on observation no dye was found above the glottis, thereby confirming the obstruction at the level of glottis.

\section{DISCUSSION}

Congenital high airway obstruction syndrome (CHAOS) is a very rare congenital malformation which takes place by deficient recanalization of the upper airways around the 10th week of gestation. ${ }^{1}$ In a healthy foetus, the fluid secreted by foetal lung is absorbed through the tracheobronchial tree. However, in case of any obstruction in the tracheobronchial tree, this fluid cannot be cleared. The accumulation of the foetal lung fluid results in gradual increase of intra tracheal pressure leading to enlargement of the lungs.

The enlarged lungs cause compression of the heart and great veins. Due to the compression, the heart displaced and becomes small and dysfunctional. Decreased venous return and dysfunctional cardiovascular system end in ascites and hydrops. The diaphragm flattens or inverts according to the severity of the process. ${ }^{4,5}$

The rare underlying causes of CHAOS are laryngeal agenesis, subglottic stenosis or atresia, and laryngeal webs or cysts. However, the obstruction is mostly secondary to laryngeal atresia. ${ }^{4}$ The identification of airway obstruction is a key to establish the diagnosis of CHAOS and to differentiate it from bilateral lung masses such as a bilateral congenital pulmonary airway malformation (CPAM) or other causes of extrinsic airway obstruction such as a double aortic arch. ${ }^{1}$

The main diagnostic tool for prenatal diagnosis of CHAOS is sonography which has typical findings on evaluation. As a natural conclusion of the pathological process, bilateral large hyper echoic lungs, small, 
compressed and displaced heart, flattened or inverted diaphragm, and ascites are characteristic findings on sonographic examination. Regarding the amniotic fluid index, compression of the esophagus by dilated airways may lead to polyhydramnios, as the foetal swallowing of the fluid is disrupted. On the other hand, impaired swallowing of the foetus may also cause oligohydramnios. The gestational age at the diagnosis may affect the amniotic fluid quantity. ${ }^{4}$

CHAOS is mostly sporadic, and the exact incidence is not known. The most common associated genetic disorder with CHAOS is Fraser's syndrome which is inherited by autosomal recessive form and characterized by urogenital defects, laryngeal atresia, syndactyly, and cryptophthalmos. ${ }^{4}$ CHAOS may be also a part of Cri-duChat syndrome, short-rib polydactyly syndrome, and velocardiofacial syndrome. ${ }^{6}$

Apart from this, a case of CHAOS with autosomal dominant inheritance of the father and his two affected children was reported. The point to take into consideration is the necessity of a detailed evaluation of all CHAOS suspected cases due to possibility of coexistence of any genetic syndrome and significant implications of inheritance for future pregnancies. ${ }^{4,6}$

CHAOS is most often misdiagnosed as bilateral congenital cystic adenomatoid malformation (CCAM). CCAM (especially type III) and upper airway obstruction secondary to intrinsic causes such as tracheal or laryngeal atresia or stenosis and tracheal webs similarly have bilateral uniform hyper echogenic appearance of the foetal lungs on sonographic examination. In order to make a differentiation between CHAOS and CCAM type III, the obstruction site with distal airway dilatation (present in CHAOS) and the systemic arterial supply (present in CCAM type III) must be clearly seen. Congenital high airway obstruction syndrome should be also differentiated from extrinsic causes of trachealaryngeal obstruction. ${ }^{4}$

Some of these extrinsic causes are lymphatic malformation, vascular rings like double aortic arch., ${ }^{4,5}$ Some intrauterine and intrapartum treatment options are available to manage this condition. It is possible that earlier foetoscopic bronchoscopy may decompress the upper airway and allow an efficient functional lung development. ${ }^{6,7}$

\section{Scope}

The only available postnatal treatment option to sustain the survival of the foetuses with CHAOS is ex utero intrapartum treatment (EXIT) with tracheal intubation and safe airway control.

The EXIT procedure is performed using high doses of inhaled halogenated agents to facilitate uterine relaxation during caesarean section. The foetus remains hemo- dynamically stable during the procedure, as the uteroplacental circulation is maintained. This procedure requires multidisciplinary approach which needs the cooperation of obstetricians, paediatric surgeons, anaesthesiologists and neonatologists. ${ }^{7-10}$

\section{CONCLUSION}

To conclude, recognition of this syndrome is very essential as it usually results in stillbirth or death shortly after delivery. An antenatal ultra-sonographic imaging shows typical finding which helps us to diagnose this entity earliest and to provide the mother an option of termination of pregnancy in view of its virtually lethal post-natal outcome. Perinatal management of CHAOS namely EXIT treatment is in budding stage.

Funding: No funding sources Conflict of interest: None declared

Ethical approval: Not required

\section{REFERENCES}

1. Rahman T, Khalequezzaman S, Ahsan S, Alam J, Sarker MK. A case report of congenital high airway obstruction syndrome (CHAOS) caused by complete laryngeal obstruction. Pulse. 2015;7(1):38-41.

2. Joshi P, Satija L, George RA, Chatterjee S, D'Souza J, Raheem A. Congenital high airway obstruction syndrome-antenatal diagnosis of a rare case of airway obstruction using multimodality imaging. MJAFI. 2012;68(1):78-80..

3. Hedrick MH, Ferro MM, Filly RA, Flake AW, Harrison MR, Adzick NS. Congenital high airway obstruction syndrome (CHAOS): a potential for perinatal intervention. J Pediatr Surg. 1994;29:271-4.

4. Ulkumen BA, Pala HG, Nese N, Tarhan S, Hindaw YB. Prenatal diagnosis of congenital high airway obstruction syndrome: report of two cases and brief review of the literature. Case Rep Obstet Gynecol. 2013:28974.

5. Mundkur A, Nayak SS, Vasudeva A, Katta GM, Kumar P. Congenital high airway obstruction syndrome presenting as nonimmune hydrops in a 19week fetus. Int J Infertil Fetal Med. 2016;7(3):105-8.

6. Aslan H, Ekiz A, Acar DK, Aydiner B, Kaya B, Sezer S. Prenatal diagnosis of congenital high airway obstruction syndrome (CHAOS). Five case report. Med Ultrason. 2015;17(1):115-8.

7. Sndeep J, Divyashree, Saket RK, Singh P. Congenital High Airway Obstruction Syndrome (CHAOS): a rare fetal malformation diagnosed on antenatal ultrasonography. Int $\mathbf{J}$ Clin Med Imaging. 2015;2(8):1000358.

8. Naikwadi A, Rege RN. Congenital high airway obstruction: case report with imaging features. JMSCR. 2016;4(11).

9. Sharma R, Dey AK, Alam S, Mittal K, Thakkar H. A series of congenital high airway obstruction 
syndrome: classic imaging findings. J Clin Diag Res. 2016;10(3):TD07-9.

10. Cho HJ, Kim YM, Lee HY, Won HS. A case of congenital high airway obstruction syndrome caused by complete tracheal obstruction with associated anomalies. Korean J Obstet Gynecol. 2012;55(2):115-8
Cite this article as: Kanchana MP, Sellamuthu MP, Mahendran R. A rare case report of congenital high airway obstruction syndrome presenting in a 23 weeks foetus. Int J Reprod Contracept Obstet Gynecol 2018;7:1277-80. 